\title{
Function computation via subspace coding
}

\author{
Nikhil Karamchandani* $^{*}$ Lorenzo Keller ${ }^{\dagger}$ \\ Christina Fragouli ${ }^{\dagger}$ Massimo Franceschetti* \\ *Dept. of Electrical and Computer Engineering \\ UCSD, USA \\ ${ }^{\dagger}$ School of Computer and Communication Sciences \\ EPFL, Switzerland
}

\{Email : nikhil@ucsd.edu, lorenzo.keller@epfl.ch, christina.fragouli@epfl.ch, massimo@ece.ucsd.edu\}

\begin{abstract}
This paper considers function computation in a network where intermediate nodes perform randomized network coding, through appropriate choice of the subspace codebooks at the source nodes. Unlike traditional network coding for computing functions, that requires intermediate nodes to be aware of the function to be computed, our designs are transparent to the intermediate node operations.
\end{abstract}

\section{INTRODUCTION}

In sensor networks, the need for energy efficiency has stimulated research efforts towards in-network aggregation and function computation, see for example [1], [2]. Recent work [3], [4] has also pointed out the need to have simple coding schemes, since "systems are hard to develop and debug". They advocate a solution where most nodes in the network perform the same operations regardless of the function to be computed, and the onus of guaranteeing successful computation is on a few special nodes that are allowed to vary their operation.

Motivated by the above considerations, we consider the problem of computing functions in a network where multiple sources are connected to a single sink via relays. The sources may have several different possible codebooks, and can select which one to employ depending on the function to be computed. Given a certain target function, each source transmits a codeword corresponding to its observed message. The relay nodes, however, perform randomized network coding [5] irrespective of the target function, i.e., source codewords are randomly combined and forwarded towards the sink, using linear coefficients that are unknown to both the sources and the sink. The sink then proceeds to evaluate the target function of the source messages.

Following [6]-[8], we use subspace coding for computing functions in our network model. Given a target function, we assume that each source uses a codebook consisting of subspaces. Each source message is associated with a corresponding subspace. When a source generates a message, it injects the basis vectors of the corresponding subspace into the network as codewords. The network operation is abstracted by assuming that the sink collects enough linear combinations of the source codewords to learn the joint span of the injected subspaces. Given this information, the sink then attempts to compute the target function of the source messages. Our objective is to design codebooks which minimize the number of symbols each source needs to transmit, while guaranteeing successful function computation by the sink.

Thus, we envision a network architecture where intermediate network nodes always perform the same operations for the information transfer, which leads to a simple implementation. At the same time, the sink has the flexibility to utilize the network to learn different functions of the source data by informing the source nodes to employ the corresponding codebooks.

The paper is organized as follows. Section II presents the problem formulation. In Section III, we present a simple scheme to compute the identity function, i.e., to reconstruct all the source messages, and then describe a class of "hard" functions for which it is optimal (in an order sense) to first compute the identity and then compute the function value. We continue by designing near-optimal coding schemes for some "easy" functions, i.e., functions which can be computed by transmitting less symbols by the sources than what is required to compute the identity: these are the T-threshold, maximum and $K$-largest values functions considered Section IV. In Section V-A, we present a lower bound on the number of symbols each source needs to transmit to evaluate an arbitrary function, and a constructive scheme to evaluate arbitrary functions.

\section{Problem Formulation}

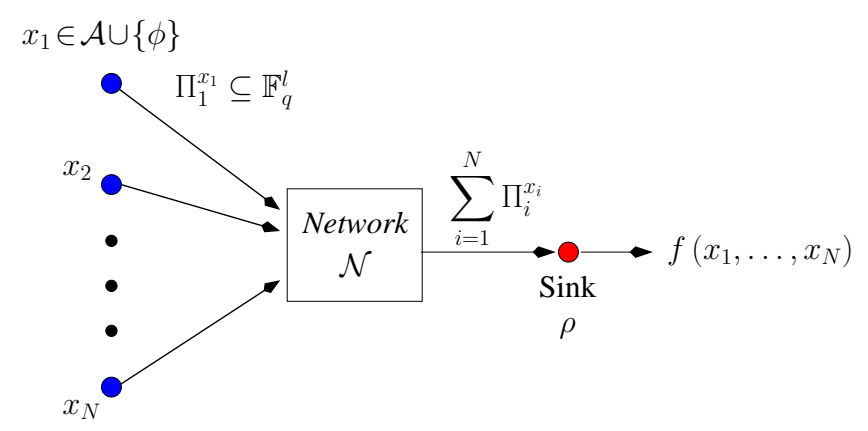

We consider a set of $N$ sources $\sigma_{1}, \sigma_{2}, \ldots, \sigma_{N}$ connected to a sink $\rho$ via a network $\mathcal{N}$. Each source $\sigma_{i}$ is either inactive or observes a message $x_{i} \in \mathcal{A}$, where $\mathcal{A}$ is a finite alphabet. For ease of notation, when a source $\sigma_{i}$ is inactive we will set $x_{i}=\phi$. The sink needs to compute a target function $f$ of the source messages, where $f$ is of the form

$$
f:(\mathcal{A} \cup\{\phi\})^{N} \longrightarrow \mathcal{B} .
$$

We consider operation using subspace coding. The network works as follows.

- At each source, every alphabet symbol is mapped to a subspace, which serves as the corresponding codeword. 
Thus, each source $\sigma_{i}$ has an associated codebook $\mathcal{C}_{i}=$ $\left\{\pi_{i}^{j}\right\}_{j \in \mathcal{A}}$ where $\pi_{i}^{j}$ is a $d$-dimensional subspace ${ }^{1}$ of an $l$-dimensional vector space $\mathbb{F}_{q}^{l}$ where $d, l \geq 1$ are design parameters. When the source $\sigma_{i}$ is active and observes a message $x_{i} \in \mathcal{A}$, it injects into the network $\mathcal{N}$ a set of $d$ vectors from $\mathbb{F}_{q}^{l}$ which span the subspace $\pi_{i}^{x_{i}}$. When the source is $\sigma_{i}$ inactive, it does not make any transmissions and hence we set $\pi_{i}^{\phi}=\emptyset$.

- The sink $\rho$ receives from the network $\mathcal{N}$ a set of vectors from $\mathbb{F}_{q}^{l}$ which span the union of the input subspaces ${ }^{2}$, i.e., $\rho$ observes $\sum_{i=1}^{N} \pi_{i}^{x_{i}}$.

- The sink uses the received information to compute the value of $f\left(x_{1}, x_{2}, \ldots, x_{N}\right)$.

A $(d, l)$ feasible code for computing $f$ is a collection of codebooks $\left\{\mathcal{C}_{1}, \mathcal{C}_{2}, \ldots, \mathcal{C}_{N}\right\}$ such that each $\pi_{i}^{j}$ in the codebooks is a $d$-dimensional subspace of $\mathbb{F}_{q}^{l}$ and the sink can compute the value of $f\left(x_{1}, x_{2}, \ldots, x_{N}\right)$ for any choice of input messages $x_{1}, x_{2}, \ldots, x_{N}$ where each $x_{i} \in \mathcal{A} \cup\{\phi\}$.

For a $(d, l)$ feasible code for computing $f$, each source transmits at most $d \cdot l$ symbols from $\mathbb{F}_{q}$, and we thus consider the associated cost to be $d \cdot l$. Our code design seeks to achieve $\mathcal{E}_{\min }(f)=\inf \{d \cdot l: \exists \mathrm{a}(d, l)$ feasible code for computing $f\}$. We will denote the dimension of any subspace $\pi$ by $\operatorname{dim}(\pi)$. Also, for any vector $\mathbf{x}$, the $j$-th component will be denoted by $(\mathbf{x})_{j}$. Consider a set of indices $I=\left(i_{1}, i_{2}, \ldots, i_{|I|}\right) \subseteq$ $\{1,2, \ldots, N\}$. For any $\mathbf{a}=\left(a_{1}, a_{2}, \ldots, a_{|I|}\right) \in(\mathcal{A} \cup$ $\{\phi\})^{|I|}$ and any vector $\mathbf{x} \in(\mathcal{A} \cup\{\phi\})^{N}$, let $\mathbf{x}(I, \mathbf{a})=$ $\left(x_{1}, x_{2}, \ldots, x_{N}\right)$ denote a vector which is obtained from $\mathbf{x}$ by substituting the components corresponding to the index set $I$ with values from the vector $\mathbf{a}$ and retaining all the other components. That is, for each $j \in\{1,2, \ldots,|I|\}$, $(\mathbf{x}(I, \mathbf{a}))_{i_{j}}=(\mathbf{a})_{j}$ and for each $k \notin I,(\mathbf{x})_{k}=(\mathbf{x}(I, \mathbf{a}))_{k}$. We conclude this section with a lemma that is often used in the subsequent sections.

Lemma II.1. For any collection $\pi_{1}, \pi_{2}, \ldots, \pi_{K} \subseteq \mathbb{F}_{q}^{l}$ of $d$ dimensional subspaces, let

$$
\pi_{i} \nsubseteq \sum_{j<i} \pi_{j} \quad \forall i \in\{1,2, \ldots, K\} .
$$

Then $d \cdot l \geq K$.

Proof: (1) implies that there exists a collection of $K$ linearly independent vectors $\mathbf{v}_{\mathbf{1}}, \mathbf{v}_{\mathbf{2}}, \ldots, \mathbf{v}_{\mathbf{K}} \in \mathbb{F}_{q}^{l}$ such that $\mathbf{v}_{\mathbf{i}} \in \pi_{i}$ for every $i \in\{1,2, \ldots, K\}$. This implies that $l \geq K$, the result then follows.

\section{FUNCTIONS WHICH ARE MAXIMALLY HARD TO COMPUTE}

Any target function can be computed by first reconstructing all the source messages at the sink (i.e., computing the

\footnotetext{
${ }^{1}$ Although restricting our code design to subspaces of equal dimension may not always be optimal, it significantly simplies the design, and is a standard approach in the literature [6], [9].

${ }^{2}$ The union of two subspaces $\pi_{1}, \pi_{2}$ is defined as $\pi_{1}+\pi_{2}=\{\mathbf{x}+\mathbf{y}$ : $\left.\mathbf{x} \in \pi_{1}, \mathbf{y} \in \pi_{2}\right\}$.
}

identity function $\left.f\left(x_{1}, x_{2}, \ldots, x_{N}\right)=\left(x_{1}, x_{2}, \ldots, x_{N}\right)\right)$ and then deriving the function value. Hence, the following lemma provides an upper bound on $d \cdot l$ for any function $f$.

Lemma III.1. There exists a $(d, l)$ feasible code for computing the identity function such that

$$
d \cdot l=N+\left\lceil\log _{q}|\mathcal{A}|\right\rceil .
$$

Proof: It is easy to see that this can be achieved simply by using coding vectors of length $N$, where source $i$ for example uses the basis vector $\mathbf{e}_{\mathbf{i}}$ as its coding vector and appends this to the information packet that consists of $\left\lceil\log _{q}|\mathcal{A}|\right\rceil$ symbols.

Consider the case $N \geq \log _{q}|\mathcal{A}|$. Next, we present a class of functions for which $d \cdot l$ is required to grow linearly with respect to the number of sources $N$. Thus, the number of transmissions that each source makes for the computation of such functions is almost the same (in the order sense) as that required to reconstruct all the source messages. For any vector $\mathbf{x} \in(\mathcal{A} \cup\{\phi\})^{N}$, let $I_{\mathbf{x}}$ denote the index set corresponding to the components which are not $\phi$. Then, consider a target function $f$ which satisfies the following property with some constant $\alpha \in(0,1]$.

Function property $\mathbf{P}(\alpha)$ : There exists a vector $\mathbf{x}^{*}=$ $\left(x_{1}^{*}, x_{2}^{*}, \ldots, x_{N}^{*}\right)$ with $\left|I_{\mathbf{x}^{*}}\right| \geq \alpha N$ such that for each $k \in I_{\mathbf{x}^{*}}$,

$$
f\left(\mathbf{x}^{*}(\{k\}, \phi)\right) \neq f\left(\mathbf{x}^{*}\right) .
$$

This implies that the function value is sensitive to whether any specific source $\sigma_{k}$ is active or not.

\section{Example III.2.}

- The identity function satisfies property $\mathbf{P}(1)$ by choosing each $x_{i}^{*}$ equal to any element of the alphabet $\mathcal{A}$.

- The arithmetic sum function satisfies property $\mathbf{P}(1)$ by choosing each $x_{i}^{*}$ equal to some non-zero element of the alphabet $\mathcal{A}$.

- The parity function ( $\mathcal{A}=\{0,1\}$ ) satisfies property $\mathbf{P}(1)$ by choosing each $x_{i}^{*}$ equal to 1 .

- The majority function ( $\mathcal{A}=\{0,1\}$ ) satisfies property $\mathbf{P}(1 / 2)$ by choosing the first $N / 2 x_{i}^{*}$ 's equal to 1 and the rest equal to 0 .

Lemma III.3. Let $f$ be a function which satisfies the property $\mathbf{P}(\alpha)$. Then,

$$
\mathcal{E}_{\min }(f) \geq \alpha N \text {. }
$$

Proof: From (2), any $(d, l)$ feasible code for computing the function $f$ must satisfy the following condition. For each $k \in I_{\mathbf{x}^{*}}$,

$$
\pi_{k}^{x_{k}^{*}}+\sum_{j \neq k} \pi_{j}^{x_{j}^{*}} \neq \sum_{j \neq k} \pi_{j}^{x_{j}^{*}} \Longrightarrow \pi_{k}^{x_{k}^{*}} \nsubseteq \sum_{j \neq k} \pi_{j}^{x_{j}^{*}} .
$$

Since $\left|I_{\mathbf{x}^{*}}\right| \geq \alpha N$, the proof then follows from Lemma II.1.

Comment : Lemma V.4 provides a general lower bound on $\mathcal{E}_{\text {min }}(f)$ for arbitrary functions. Functions for which the lower bound is of the same order as $N+\left\lceil\log _{q}|\mathcal{A}|\right\rceil$ are also 
maximally hard to compute.

\section{BOUNDS FOR SPECIFIC FUNCTIONS}

\section{A. T-threshold Function}

Let $\mathcal{A}=\{1\}$. The $T$-threshold function is defined as ${ }^{3}$

$$
f\left(x_{1}, x_{2}, \ldots, x_{N}\right)= \begin{cases}1 & \text { if } x_{1}+x_{2}+\ldots+x_{N} \geq T \\ 0 & \text { otherwise. }\end{cases}
$$

Lemma IV.1. There exists a $(d, l)$ feasible code for computing the $T$-threshold function with $T<N / 2$, such that

$$
d \cdot l \leq O\left(N H_{q}\left(\frac{T}{2 N}\right)\right) .
$$

Proof: Consider the following scheme.

A $(1, l)$ code for the T-threshold function :

- Let $\mathbf{H}$ be the $l \times N$ parity check matrix of a binary code with minimum distance $d_{\text {min }}=T+1$.

- Source $\sigma_{i}$ uses $C_{i}=\left\{\mathbf{h}_{\mathbf{i}}\right\}$, where $\mathbf{h}_{\mathbf{i}}$ is a column of $\mathbf{H}$.

- If the dimension of the subspace that the sink receives is less than $T$, it outputs 0 . Otherwise, it outputs 1 .

The above scheme uses a $l \times N$ parity check matrix of a binary code with minimum distance $d_{\min }=T+1$. From [10], there exists such a matrix with

$$
l \leq O\left(N H_{q}\left(\frac{T}{2 N}\right)\right) .
$$

Since all sources transmit one-dimensional subspaces, the result follows.

Comment : For a constant $T, O\left(N H_{q}\left(\frac{T}{2 N}\right)\right)=$ $O\left(T \log _{q} N\right)$. Thus, while computing the identity function requires $d \cdot l$ to grow linearly with the number of sources $N$, the $T$-threshold function requires only logarithmic growth.

We have the following matching lower bound.

Lemma IV.2. For the $T$-threshold function $f$ with $T<N / 2$,

$$
\mathcal{E}_{\text {min }}(f) \geq \frac{N}{2} H_{q}\left(\frac{T}{2 N}\right) .
$$

where $H_{q}$ is the q-ary entropy function.

Proof: Consider two possible input vectors $\left(x_{1}, x_{2}, \ldots, x_{N}\right)$ and $\left(y_{1}, y_{2}, \ldots, y_{N}\right)$ such that

$$
\begin{aligned}
& x_{i}=1 \forall i \in\{1,2, \ldots, T\} \text { and } x_{i}=\phi \text { otherwise } \\
& y_{i}=1 \forall i \in\{2,3, \ldots, T\} \text { and } y_{i}=\phi \text { otherwise } .
\end{aligned}
$$

Note that

$$
1=f\left(x_{1}, x_{2}, \ldots, x_{N}\right) \neq f\left(y_{1}, y_{2}, \ldots, y_{N}\right)=0
$$

and hence it is necessary for any $(d, l)$ feasible code for

\footnotetext{
${ }^{3}$ For any integer $a$, we set $a+\phi=a$. Thus, the function computes whether the number of active sources is at least $T$ or not.
}

computing $f$ that

$$
\pi_{1}^{1}+\sum_{i=2}^{T} \pi_{i}^{1} \neq \sum_{i=2}^{T} \pi_{i}^{1} \Longrightarrow \pi_{1}^{1} \nsubseteq \sum_{i=2}^{T} \pi_{i}^{1} .
$$

The same argument can be extended to get the following necessary condition. For any subset $\left(i_{1}, i_{2}, \ldots, i_{T}\right)$ of $\{1,2, \ldots, N\}$,

$$
\pi_{i_{j}}^{1} \not \sum_{k \neq j} \pi_{i_{k}}^{1} \text { for every } j \in\{1,2, \ldots, T\} .
$$

Denote the basis vectors for any $\pi_{i}^{1}$ by $\left(\mathbf{v}_{\mathbf{i}}^{\mathbf{1}}, \mathbf{v}_{\mathbf{i}}^{\mathbf{2}}, \ldots, \mathbf{v}_{\mathbf{i}}^{\mathbf{d}}\right)$. Construct a vector $\mathbf{v}_{\mathbf{i}}^{*}$ of length $d \cdot l$ by concatenating the $d$ basis vectors. From the necessary condition on the subspaces $\pi_{1}^{1}, \pi_{2}^{1}, \ldots, \pi_{N}^{1}$, any collection of $T$ vectors from $\mathbf{v}_{\mathbf{1}}^{*}, \mathbf{v}_{\mathbf{2}}^{*}, \ldots, \mathbf{v}_{\mathbf{N}}^{*}$ are linearly independent. A $d \cdot l \times N$ matrix with the vectors $\mathbf{v}_{1}^{*}, \mathbf{v}_{\mathbf{2}}^{*}, \ldots, \mathbf{v}_{\mathbf{N}}^{*}$ as columns corresponds to the parity check matrix for a linear code of length $N$ and minimum distance at least $T+1$. Using the bounds in [10], for $T<N / 2$ we have

$$
d \cdot l \geq N H_{q}\left(\frac{T}{2 N}\right)-\frac{1}{2} \log _{q}\left(4 T\left(1-\frac{T}{2 N}\right)\right) .
$$

The result then follows since

$$
\frac{1}{2} \log _{q}\left(4 T\left(1-\frac{T}{2 N}\right)\right) \leq \frac{N}{2} H_{q}\left(\frac{T}{2 N}\right) .
$$

For $N \leq 11$, (3) can be verified numerically. Let $N \geq 12$. Then (3) holds if we show that for every $1 \leq T<N / 2$,

$$
\begin{gathered}
N \cdot \frac{T}{2 N} \ln \left(\frac{2 N}{T}\right) \geq \ln (4 T) \text { or equivalently, } \\
T \ln \left(\frac{2 N}{T}\right)-2 \ln (4 T) \geq 0 .
\end{gathered}
$$

For $T=1$, (4) holds since $N \geq 8$. Differentiating the lefthand side of (4) with respect to $T$, we get

$$
\ln (2 N)-\ln (T)-1-\frac{2}{T}
$$

which is greater than zero since $N \geq 12$ and $T \leq N / 2$. Thus, (4) is true for every $1 \leq T<N / 2$ and thus (3) holds.

\section{B. Maximum Function}

Lemma IV.3. There exists a $(d, l)$ feasible code for computing the maximum function such that

$$
\left.d \cdot l \leq \min \left\{|\mathcal{A}|, N+\left\lceil\log _{q}|\mathcal{A}|\right\rceil\right)\right\} .
$$

Proof: Consider the following two schemes for computing the maximum function ${ }^{4}$.

- $A(1,|\mathcal{A}|)$ scheme : Let $\mathbf{v}_{\mathbf{1}}, \mathbf{v}_{\mathbf{2}}, \ldots, \mathbf{v}_{|\mathcal{A}|}$ be linearly independent vectors of length $|\mathcal{A}|$ each. For every source $\sigma_{i}$, let $\mathcal{C}_{i}=\left(\mathbf{v}_{\mathbf{1}}, \mathbf{v}_{\mathbf{2}}, \ldots, \mathbf{v}_{|\mathcal{A}|}\right)$. This scheme has $d \cdot l=|\mathcal{A}|$.

- $A\left(1, N+\left\lceil\log _{q}|\mathcal{A}|\right\rceil\right)$ scheme : We can compute the identity function with $d \cdot l=N+\left\lceil\log _{q}|\mathcal{A}|\right\rceil$ and hence

\footnotetext{
${ }^{4}$ For any $a \in \mathcal{A}$, we set $\max \{a, \phi\}=a$
} 
can compute the maximum function also. This scheme is useful if $\mathcal{A} \geq N$.

Comment : Thus when $|\mathcal{A}| \ll N$, the first scheme is much more efficient than reconstructing all the source messages.

Lemma IV.4. For the maximum target function $f$,

$$
\mathcal{E}_{\text {min }}(f) \geq \min \{|\mathcal{A}|, N\} .
$$

Proof: Let $\mathcal{A}=\left(a_{1}, a_{2}, \ldots, a_{|\mathcal{A}|}\right)$ be an ordered set (in increasing order) and let $M=\min \{N,|\mathcal{A}|\}$. Consider two possible input vectors $\left(x_{1}, x_{2}, \ldots, x_{N}\right)$ and $\left(y_{1}, y_{2}, \ldots, y_{N}\right)$ such that

$$
\begin{aligned}
& x_{i}=a_{i} \forall i \in\{1,2, \ldots, M\} \text { and } x_{i}=\phi \text { otherwise } \\
& y_{i}=a_{i} \forall i \in\{1,2, \ldots, M-1\} \text { and } y_{i}=\phi \text { otherwise } .
\end{aligned}
$$

Note that

$$
M=f\left(x_{1}, x_{2}, \ldots, x_{N}\right) \neq f\left(y_{1}, y_{2}, \ldots, y_{N}\right)=M-1
$$

and hence any $(d, l)$ feasible code for computing $f$ must satisfy the following condition.

$$
\sum_{i=1}^{M-1} \pi_{i}^{a_{i}}+\pi_{M}^{a_{M}} \neq \sum_{i=1}^{M-1} \pi_{i}^{a_{i}} \Longrightarrow \pi_{M}^{a_{M}} \nsubseteq \sum_{i=1}^{M-1} \pi_{i}^{a_{i}}
$$

The same argument can be extended to get the following necessary condition. For any subset $\left(i_{1}, i_{2}, \ldots, i_{M}\right)$ of $\{1,2, \ldots, N\}$ and any ordered subset (in increasing order) $\left(a_{j_{1}}, a_{j_{2}}, \ldots, a_{j_{M}}\right)$ of $\mathcal{A}$,

$$
\pi_{i_{k}}^{a_{j_{k}}} \nsubseteq \sum_{m<k} \pi_{i_{m}}^{a_{j_{m}}}
$$

Then the result follows from Lemma II.1.

\section{K-largest Values Function}

Let $\mathcal{A}=\left(a_{1}, a_{2}, \ldots, a_{|\mathcal{A}|}\right)$ be an ordered set (in increasing order). For any given input vector $\left(x_{1}, x_{2}, \ldots, x_{N}\right)$, let $\left(\hat{x}_{1}, \hat{x}_{2}, \ldots, \hat{x}_{N}\right)$ denote the vector which is a permutation of the input vector and satisfies $\hat{x}_{i} \geq \hat{x}_{i+1}$ for each $i$. Then the $K$-largest values function is given by

$$
f\left(x_{1}, x_{2}, \ldots, x_{N}\right)=\left(\hat{x}_{1}, \hat{x}_{2}, \ldots, \hat{x}_{K}\right) .
$$

Lemma IV.5. There exists a $(d, l)$ feasible code for computing the $K$-largest values function with $K<N / 2$, such that

$$
d \cdot l \leq|\mathcal{A}| \cdot O\left(N H_{q}\left(\frac{K}{2 N}\right)\right) .
$$

Proof: Consider the following scheme.

$A(1, l)$ code for $K$-largest values function

- Let $\mathbf{H}$ be the $(l /|\mathcal{A}|) \times N$ parity check matrix of a binary code with minimum distance $K+1$.

- If source $\sigma_{i}$ takes value $a_{j}$ from the alphabet $\mathcal{A}$, then it transmits a vector which is all zero except the $(j-1) \times(l /|\mathcal{A}|)+1$ to $j \times(l /|\mathcal{A}|)$ elements, which take values from the $i$-th column of $\mathbf{H}$.

- Each vector in the union subspace $\Pi$ that the sink receives is parsed into $|\mathcal{A}|$ sub-vectors of length $l /|\mathcal{A}|$.

- Let $\Pi_{j} \subseteq \mathbb{F}_{q}^{l /|\mathcal{A}|}$ denote the subspace spanned by collecting the $j$-th sub-vector of each vector in $\Pi$.

- Let the number of sources which observe value $a_{j}$ be $N_{j}$. If $N_{j} \leq K$, then $\operatorname{dim}\left(\Pi_{j}\right)=N_{j}$.

- Thus by calculating $\operatorname{dim}\left(\Pi_{|\mathcal{A}|}\right), \operatorname{dim}\left(\Pi_{|\mathcal{A}|-1}\right) \ldots$, the sink can compute the $K$ largest values.

Again from [10], there exists a parity check matrix such that

$$
\frac{l}{|\mathcal{A}|} \leq O\left(N H_{q}\left(\frac{K}{2 N}\right)\right) .
$$

Since all sources transmit one-dimensional subspaces, the result follows.

Comment : Again, for constant $|\mathcal{A}|$ and $K, d \cdot l$ only grows logarithmically with the number of sources $N$.

Lemma IV.6. For the $K$-largest values target function $f$ with $K<N / 2$,

$$
\mathcal{E}_{\text {min }}(f) \geq \frac{N}{2} H_{q}\left(\frac{K}{2 N}\right) .
$$

Proof: If the receiver can correctly compute the $K$-largest values, then it can also deduce if the number of active sources is greater than $K$ or not. Thus, it can also compute the $T$ threshold function with the threshold $T=K$. The result then follows from Lemma IV.2.

\section{ARbitrary FunCtions}

\section{A. A general lower bound}

We begin with the following lemma.

Lemma V.1. The number of subspaces of dimension $d$ in $\mathbb{F}_{q}^{l}$ is at most $4 q^{d(l-d)}$ [6, Lemma 4].

Consider the following function property. Function property $\mathbf{P}$ : For each source $\sigma_{k}$ and any $a, b \in \mathcal{A}$, there exists $\mathbf{x}$ such that

$$
f(\mathbf{x}(\{k\}, a)) \neq f(\mathbf{x}(\{k\}, b)) .
$$

Examples: The identity function and arithmetic sum function satisfy property $\mathbf{P}$. We have the following simple lower bound.

Lemma V.2. For any target function $f$ which satisfies property 
$\mathbf{P}$

$$
\mathcal{E}_{\text {min }}(f) \geq \log _{q} \frac{|\mathcal{A}|}{4} .
$$

Proof: For any $(d, l)$ feasible code for computing $f$, each source must assign a distinct $d$-dimensional subspace to each $a \in \mathcal{A}$. From Lemma V.1, we have

$$
\begin{aligned}
& 4 q^{d(l-d)} \geq|\mathcal{A}| \\
& \Rightarrow d \cdot l \geq \log _{q} \frac{|\mathcal{A}|}{4} .
\end{aligned}
$$

Consider the following general lemma.

Lemma V.3. Let $\pi \subseteq \mathbb{F}_{q}^{l}$ be a subspace of dimension $d_{1}$. Let $\pi_{1}, \pi_{2}, \ldots, \pi_{K} \subseteq \mathbb{F}_{q}^{l}$ be $d_{2}$-dimensional subspaces such that for every $i \neq j, \pi+\pi_{i} \neq \pi+\pi_{j}$. Then,

$$
l \geq \max \left\{\frac{\sqrt{\log _{q}(K-1)}}{3}, \frac{\log _{q}(K-1)}{3 d_{2}}\right\} .
$$

Proof: Denote the complement subspace of $\pi$ by $\bar{\pi}$ $\left(\pi \cap \bar{\pi}=\phi, \pi+\bar{\pi}=\mathbb{F}_{q}^{l}\right)$. Let $<\mathbf{b}_{1}, \ldots, \mathbf{b}_{\mathbf{d}_{1}}>$ be a basis of $\pi$ and $<\mathbf{b}_{\mathbf{d}_{1}+\mathbf{1}}, \ldots, \mathbf{b}_{\mathbf{l}}>$ be a basis of $\bar{\pi}$ so that together they span $\mathbb{F}_{q}^{l}$. Now let $\left\langle\mathbf{c}_{1}, \ldots, \mathbf{c}_{\mathbf{d}_{2}}\right\rangle$ denote the basis for any subspace $\pi_{i}$. Then each $c_{i}$ can be expressed as a linear combination of the $\mathbf{b}_{\mathbf{i}}$ 's, that is, $c_{i}=\alpha_{1, i} \mathbf{b}_{\mathbf{1}}+\ldots+\alpha_{l, i} \mathbf{b}_{\mathbf{1}}$. Thus, $\pi+\pi_{i}$ is a subspace spanned by $<\mathbf{b}_{\mathbf{1}}, \ldots, \mathbf{b}_{\mathbf{d}_{\mathbf{1}}}, \sum_{i=1}^{l} \alpha_{i, 1} \mathbf{b}_{\mathbf{i}}, \ldots, \sum_{i=1}^{l} \alpha_{i, d} \mathbf{b}_{\mathbf{i}}>$. This is equivalent to the subspace spanned by $<$ $\mathbf{b}_{\mathbf{1}}, \ldots, \mathbf{b}_{\mathbf{d}_{\mathbf{1}}}, \sum_{i=d_{1}+1}^{l} \alpha_{i, 1} \mathbf{b}_{\mathbf{i}}, \ldots, \sum_{i=d_{1}+1}^{l} \alpha_{i, d} \mathbf{b}_{\mathbf{i}}>$, where the last $d$ vectors are a linear combination of vectors in $\bar{\pi}$. Therefore for each subspace $\pi_{i}$, there exists a subspace $\widetilde{\pi}_{i} \subseteq \bar{\pi}$ such that $\pi+\pi_{i}=\pi+\widetilde{\pi}_{i}$ and $\widetilde{\pi}_{i} \cap \pi=\phi$. Then for every $i \neq j, \widetilde{\pi}_{i} \neq \widetilde{\pi}_{j}$ since $\pi+\widetilde{\pi}_{i} \neq \pi+\widetilde{\pi_{j}}$. Further, each $\widetilde{\pi}_{i}$ has dimension at most $d_{2}$. Note that the dimension of $\pi$ is $l-d_{1}$ and each subspace $\widetilde{\pi}_{i}$ is a subspace of $\Pi$. Since there are $K$ distinct $\widetilde{\pi_{i}}$ 's, we have from Lemma V.1 that

$$
1+4 \cdot \sum_{j=1}^{\min \left\{l-d_{1}, d_{2}\right\}} q^{j\left(l-d_{1}-j\right)} \geq K .
$$

Then, we have

$$
\begin{aligned}
& 4 \cdot \sum_{j=1}^{l-d_{1}} q^{j\left(l-d_{1}-j\right)} \geq K-1 \\
& \Rightarrow 4\left(l-d_{1}\right) \cdot q^{\left(\frac{l-d_{1}}{2}\right)^{2} \geq K-1} \\
& \Rightarrow \log _{q}\left(4\left(l-d_{1}\right)\right)+\left(\frac{l-d_{1}}{2}\right)^{2} \geq \log _{q}(K-1) .
\end{aligned}
$$

Since $\log _{q}\left(4\left(l-d_{1}\right)\right) \leq 2\left(l-d_{1}\right)^{2}$, we have

$$
\begin{aligned}
& 3\left(l-d_{1}\right)^{2} \geq \log _{q}(K-1) \\
& \Rightarrow l \geq \frac{\sqrt{\log _{q}(K-1)}}{3} .
\end{aligned}
$$

From (5), we also have

$$
\begin{aligned}
& 4 \cdot \sum_{j=1}^{d_{2}} q^{j\left(l-d_{1}-j\right)} \geq K-1 \\
& \Rightarrow 4 d_{2} \cdot q^{\hat{d}\left(l-d_{1}-\hat{d}\right)} \geq K-1 \text { with } \hat{d}=\underset{j \in\left\{1, d_{2}\right\}}{\operatorname{argmax}} q^{j\left(l-d_{1}-j\right)} \\
& \Rightarrow \log _{q}\left(4 d_{2}\right)+\hat{d}\left(l-d_{1}-\hat{d}\right)+\geq \log _{q}(K-1) .
\end{aligned}
$$

Since $\log _{q}\left(4 d_{2}\right) \leq 2 d_{2}$ and $\hat{d} \leq d_{2}$, we have

$$
\begin{aligned}
& 2 d_{2} l+d_{2} l \geq \log _{q}(K-1) \\
& \Rightarrow l \geq \frac{\log _{q}(K-1)}{3 d_{2}} .
\end{aligned}
$$

For any $\mathbf{x} \in(\mathcal{A} \cup\{\phi\})^{N}$ and $I \subseteq\{1,2, \ldots, N\}$, let

$$
R_{I}^{\mathbf{x}}(f)=\left|\left\{f(\mathbf{x}(I, \mathbf{a})): \mathbf{a} \in(\mathcal{A} \cup\{\phi\})^{|I|}\right\}\right|
$$

denote the number of distinct values that the function takes when only the arguments corresponding to $I$ are varied and all the others are held fixed according to $\mathbf{x}$. Also, for any $(d, l)$ code, any input vector $\mathbf{x} \in(\mathcal{A} \cup\{\phi\})^{N}$ and $I \subseteq\{1,2, \ldots, N\}$, let

$$
\Pi_{\mathrm{x}}^{I}=\sum_{i \in I} \pi_{i}^{x_{i}} .
$$

Lemma V.4. For any target function $f$,

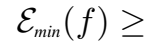

$$
\max _{\substack{I, \mathbf{x} \\ R_{I}^{\mathbf{x}}(f)>1}} \max \left\{\frac{\sqrt{\log _{q}\left(R_{I}^{\mathbf{x}}(f)-1\right)}}{3}, \frac{\log _{q}\left(R_{I}^{\mathbf{x}}(f)-1\right)}{3|I|}\right\} .
$$

Proof: Consider any $I \subseteq\{1,2, \ldots, N\}$ and any input vector $\mathbf{x}$. For any $\mathbf{a}, \mathbf{b} \in(\mathcal{A} \cup\{\phi\})^{|I|}$, if $f(\mathbf{x}(I, \mathbf{a})) \neq$ $f(\mathbf{x}(I, \mathbf{b}))$, then any $(d, l)$ feasible code should satisfy the following condition.

$$
\begin{gathered}
\sum_{j \in\{1, \ldots,|I|\}} \pi_{i_{j}}^{a_{j}}+\sum_{i \in I^{c}} \pi_{i}^{x_{i}} \neq \sum_{j \in\{1, \ldots,|I|\}} \pi_{i_{j}}^{b_{j}}+\sum_{i \in I^{c}} \pi_{i}^{x_{i}} \\
\Rightarrow \Pi_{\mathbf{x}(I, \mathbf{a})}^{I}+\Pi_{\mathbf{x}}^{I^{c}} \neq \Pi_{\mathbf{x}(I, \mathbf{b})}^{I}+\Pi_{\mathbf{x}}^{I^{c}} .
\end{gathered}
$$

Note that for any $I$ and $a \in(\mathcal{A} \cup\{\phi\})^{|I|}, \operatorname{dim}\left(\Pi_{\mathbf{x}(I, \mathbf{a})}^{I}\right) \leq$ $d \cdot|I|$ since it is composed of the union of at most $|I| d$ dimensional subspaces. Then, (7) and (6) imply that there exist $R_{I}^{\mathbf{x}}(f)$ subspaces, each with dimesion at most $d \cdot|I|$, such that the union of any one of them with $\Pi_{\mathrm{x}}^{I^{c}}$ is unique. Since $I, \mathbf{x}$ were arbitrary, the result follows from Lemma V.3.

\section{Example V.5.}

- For the identity target function $f$, the above bound gives

$$
\mathcal{E}_{\text {min }}(f) \geq \frac{\log _{q}|\mathcal{A}|}{3} .
$$

- For the arithmetic sum target function $f$, we get

$$
\mathcal{E}_{\text {min }}(f) \geq \frac{\sqrt{\log _{q} N|\mathcal{A}|}}{3} .
$$


Comment : Note that when $|\mathcal{A}| \gg N$, the bounds in the above examples are better than the ones presented in previous sections.

\section{B. A general scheme for computation}

We now present a general method to compute functions under our network model. We will illustrate the method for boolean functions of the form $f:(\mathcal{A} \cup\{\phi\})^{N} \rightarrow\{0,1\}$. For a general function, the output can be considered as a string of bits and the above scheme can be used separately to compute each bit of the output.

Since $f$ has boolean output, it can be written as

$$
f\left(x_{1}, x_{2}, \ldots, x_{N}\right)=\sum_{i=1}^{s} \prod_{j=1}^{N} B_{i j}
$$

where $s$ is some integer such that $1 \leq s \leq|\mathcal{A}|^{N} ;\left\{B_{i j}\right\}$ are boolean variables such that the value of $B_{i j}$ depends only on $x_{j}$; and the sum and product represent boolean OR and AND. By taking the complement, we have

$$
\overline{f\left(x_{1}, x_{2}, \ldots, x_{N}\right)}=\prod_{i=1}^{s} \sum_{j=1}^{N} \overline{B_{i j}} .
$$

Given any input $x_{j}$, source $j$ creates a vector $v_{j}$ of length $s$ such that $i$-th component is $\overline{B_{i j}}$. Each source $j$ then sends the corresponding vector $v_{j}$ into the network and the sink collects linear combinations of these vectors. If the $i$-th component of any of the vectors in the union subspace at the sink is 1 , then a boolean variable $A_{i}$ is assigned the value 1 . This implies that

$$
A_{i}=\sum_{j=1}^{N} \overline{B_{i j}}
$$

and hence,

$$
f\left(x_{1}, x_{2}, \ldots, x_{N}\right)=\overline{\prod_{i=1}^{s} A_{i}} .
$$

Thus, we have a $(1, s)$ scheme with $d \cdot l=s$ to compute any function $f$ with binary output.

Comment : Since $d \cdot l=s$, the above scheme is efficient when the number of input vectors for which the function value is 1 (or 0 ) is much smaller than the total number of possible input vectors.

We now present an example to illustrate the above method.

Example V.6. Let $\mathcal{B}=\{1,2, \ldots, K\}$ and let the source alphabet $\mathcal{A}$ be the power set of $\mathcal{B}$, i.e, $\mathcal{A}=2^{\mathcal{B}}$. Then the set cover function is defined as

$$
f\left(x_{1}, x_{2}, \ldots, x_{N}\right)= \begin{cases}1 & \text { if } \mathcal{B} \nsubseteq \bigcup_{i=1}^{N} x_{i} \\ 0 & \text { otherwise. }\end{cases}
$$

In words, each source observes a subset of $\mathcal{B}$ and the sink needs to compute if the union of the source messages covers
$\mathcal{B}$. Define the boolean variable $\mathbb{1}_{A}$ as follows.

$$
\mathbb{1}_{A}=\left\{\begin{array}{l}
1 \quad \text { if } A \text { is true } \\
0 \quad \text { otherwise. }
\end{array}\right.
$$

Then the function $f$ can be rewritten as

$$
f\left(x_{1}, x_{2}, \ldots, x_{N}\right)=\sum_{i=1}^{K} \prod_{j=1}^{N} \mathbb{1}_{\left\{i \notin x_{j}\right\}} .
$$

Then using the scheme described in this section, the set cover function can be computed using a $(1, K)$ code with $d \cdot l=$ $\log _{2}|\mathcal{A}|=K$. This scheme is in-fact optimal in terms of the smallest possible $d \cdot l$ for any feasible code.

\section{Conclusions}

In this paper we investigated function computation in a network where intermediate nodes perform randomized network coding, through appropriate choice of the subspace codebooks at the source nodes. Unlike traditional function computation, that requires intermediate nodes to be aware of the function to be computed, our designs are transparent to the intermediate node operations. Future work includes finding tighter bounds for general functions as well as designing more efficient schemes. Another direction of research would be to relax our assumption that the sink is able to observe the joint span of the injected subspaces and allow it to only learn some subspace of the union.

\section{REFERENCES}

[1] A. Giridhar and P. R. Kumar, "Computing and communicating functions over sensor networks," IEEE Journal on Selected Areas in Communication, vol. 23, no. 4, pp. 755-764, Apr. 2005.

[2] - "Toward a theory of in-network computation in wireless sensor networks," IEEE Communications Magazine, vol. 44, no. 4, pp. 98-107, Apr. 2006.

[3] J. Paek, B. Greenstein, O. Gnawali, K. Jang, A. Joki, M. Vieira, J. Hicks, D. Estrin, R. Govindan, and E. Kohler, "The tenet architecture for tiered sensor networks," ACM Transactions on Sensor Networks (TOSN), 2009.

[4] O. Gnawali, K. Jang, J. Paek, M. Vieira, R. Govindan, B. Greenstein, A. Joki, D. Estrin, and E. Kohler, "The tenet architecture for tiered sensor networks," in Proceedings of the ACM Conference on Embedded Networked Sensor Systems (SenSys), Oct 2006, pp. 153-166.

[5] T. Ho, M. Medard, R. Koetter, D. R. Karger, M. Effros, J. Shi, and B. Leong, "A random linear network coding approach to multicast," IEEE Transactions on Information Theory, vol. 52, no. 10, pp. 44134430, Oct. 2006.

[6] R. Koetter and F. R. Kschischang, "Coding for errors and erasures in random network coding," IEEE Transactions on Information Theory, vol. 54, no. 8, pp. 3579-3591, Aug 2008.

[7] M. Jafari Siavoshani, C. Fragouli, and S. Diggavi, "Noncoherent multisource network coding," in Proceedings of the IEEE International Symposium on Information Theory (ISIT), Jul 2008, pp. 817-821.

[8] C. Fragouli, M. Jafari Siavoshani, S. Mohajer, and S. Diggavi, "On the capacity of non-coherent network coding," in Proceedings of the IEEE International Symposium on Information Theory (ISIT), Jun 2009, pp. 273-277.

[9] D. Silva, F. R. Kschischang, and R. Koetter, "A rank-metric approach to error control in random network coding," IEEE Transactions on Information Theory, vol. 54, no. 9, pp. 3951-3967, Sep 2008.

[10] L. Keller, M. Siavoshani, C. Fragouli, K. Argyraki, and S. Diggavi, "Identity aware sensor networks," in Proceedings of the IEEE Conference on Computer Communications (INFOCOM), Apr 2009, pp. 2177 2185 . 